\title{
Coalition Building: What Happens When External Facilitators Put the CBPR Principles in Practice? Ethnographic Examples From the Massachusetts HEALing Communities Study
}

\author{
Mari-Lynn Drainoni ( $\sim$ drainoni@bu.edu ) \\ Boston University \\ Galya Walt \\ Boston Medical Center \\ Linda Sprague Martinez \\ Boston University \\ Rebecca Smeltzer \\ Boston Medical Center
}

Savanna Santarpio

Boston Medical Center

Rosie Munoz-Lopez

Boston Medical Center

Craig McClay

Boston Medical Center

Lauren Keisling

Boston Medical Center

Aumani Harris

Boston Medical Center

Faizah Gillen

Boston Medical Center

Valerie El-Alfi

Boston Medical Center

Erika L. Crable

University of California, San Diego

Jane F. Carpenter

Boston Medical Center

Laura K. Barkowski

Boston Medical Center

Tracy A. Battaglia

Boston Medical Center 


\section{Research Article}

Keywords: Community Based Participatory Research Principles, Ethnographic Methods, Addiction Medicine, Opioid Use Disorder, Coalitions, Facilitation

Posted Date: March 3rd, 2022

DOI: https://doi.org/10.21203/rs.3.rs-1396187/v1

License: (c) (1) This work is licensed under a Creative Commons Attribution 4.0 International License. Read Full License 


\section{Abstract \\ Background}

The HEALing Communities Study (HCS) is a large-scale multisite study testing Community Engagement using coalition facilitation as an approach to addressing the worsening overdose crisis. Within Community Engagement, Community-Based Participatory Research (CBPR) principles guide researchers on best practices for working in partnership with communities, yet these principles have not been well researched in large complex multi-site studies.

\section{Methods}

This paper used ethnographic methods to explore how coalitions operationalized the CBPR principles during early coalition formation. Two coders independently analyzed 101 ethnographies from HCS coalition meetings in eight Massachusetts communities held between November 2019 and December 2020. Themes were developed through consensus between the coders followed by group discussions among the authorship team.

\section{Results}

We found mutual trust, shared goals, addressing power dynamics, meeting structure and attending to the sociopolitical community context could all serve as barriers or facilitators to operationalizing the CBPR principles.

\section{Conclusions}

These findings provide unique suggestions for future community-engaged multisite studies, and demonstrate the importance of research teams to mitigate inherent power imbalances by acknowledging and creating space for community ownership. The findings also highlight the value of a community engaged facilitator role to promote the CBPR principles. Strategies such as transparency, uniting over shared interests and bringing in a wide range of stakeholders were notable facilitators for operationalizing the principles.

\section{Introduction}

The ongoing opioid overdose epidemic is a major public health crisis in the United States. More than 69,710 opioid-related overdose deaths occurred in 2020 [1]. Rates of fatal and non-fatal opioid-related overdoses are expected to increase due to social isolation and limited access to treatment services related to the COVID-19 pandemic [2, 3]. Although opioid overdose is a well-documented national epidemic, drivers of opioid overdose rates are complex and vary across and within communities and 
regions. Innovative approaches tailored to local context that are also grounded in scientific theory are necessary to support community-driven efforts to reduce fatal opioid-related overdoses. Community engagement (CE) is one approach to addressing the overdose crisis. Community engaged research (CEnR) represents a continuum of approaches that emphasize participatory and cooperative co-learning between communities and academic partners. By focusing on local community capacity building and empowerment, CEnR achieves a balance between research and action [4]. Community-based participatory research (CBPR) is an exemplar methodology on the CEnR continuum that uses a core set of principles steeped in Freirean theories of popular education and liberation [5]. These core principles guide the development of equitable partnerships between the community and the research team by involving community stakeholders in the study process, and building off local expertise, experience, and unique strengths and perspectives [6]. As opposed to traditional research methods, CBPR researchers work collaboratively "with" community members who share responsibility for all elements of the study [4].

The literature identifies ten guiding principles of CBPR, including to: 1) recognize the community as the unit of identity; 2) build on strengths and resources within the community; 3 ) facilitate a collaborative, equitable partnership in all phases of the research; 4) promote co-learning and capacity-building among all partners; 5) integrate and achieve a balance between research and action to mutually benefit all partners; 6) emphasize public health problems of local relevance and ecological perspectives that recognize the multiple determinants of health and disease; 7) use a cyclical and iterative process; 8) disseminate findings to all partners and involve all partners in dissemination; 9) establish a long-term commitment to the process, and 10) address issues of race, ethnicity, racism, and social class and embrace "cultural humility" $[4,7,8,9]$. The core of these principles has remained the same in the literature, despite some variation over time.

CBPR-guided research has the ability to impact outcomes and sustainability of study efforts [10]. However, few large-scale multisite trial studies identify how the CBPR principles are demonstrated or adopted. The HEALing (Helping to End Addiction Long-term) Communities Study (HCS) is a community engaged multisite, cluster-randomized trial that draws on principles of CBPR to build or partner with existing coalitions in 67 communities across four states [11, ClinicalTrials.gov identifier NCT04111939]. The goal of the HCS is to examine the effectiveness and implementation success of the Communities that Heal Intervention (CTH), a three-component intervention based on expanding evidence-based practices (EBPs) shown to reduce opioid overdose deaths [12]. The three CTH components include: 1) community engagement with coalitions to develop and deploy comprehensive, data-driven plans for EBP selection and implementation across multiple community sectors [13]; 2) the Opioid Reduction Continuum of Care Approach (ORCCA), which consists of a menu of EBPs to be implemented in the areas of overdose education and naloxone distribution (OEND), medication for opioid use disorder (MOUD) treatment and safer opioid prescribing [14]; and 3) community-informed communication campaigns to address stigma and raise awareness about services [15].

Community engagement in the $\mathrm{CTH}$ intervention occurs within a coalition building process. Coalition building and planning engages local stakeholders from each community and those most impacted by the 
decision-making process including people who live, work, and use drugs in the community. Coalition building is found to be an effective implementation strategy for planning and implementing communitybased health interventions [16]. In the Massachusetts HCS CTH process, a "community-facing team" guides the CEnR activities. The community-facing team is led by a community engagement facilitator (CEF) who guides the coalition through the six phases of the CTH intervention [13]. This individual leads the coalition formation and development process, serves as a liaison between the community coalition members and the study, and assists the community coordinator in implementation of new interventions. The community-facing team also includes a community data manager (CDM) with statistical and epidemiological expertise who supports monitoring and implementation progress and a community clinical faculty member with subject matter expertise in addiction medicine. Each coalition also hires a coalition coordinator who manages the coalition on a day-to-day basis and is based in the community.

The CE model incorporates CBPR principles to shape the way research staff engage with community partners. In Massachusetts, training emphasizing the core principles of CBPR was required for all research staff prior to study implementation. While the CBPR guiding principles are important to fostering true community engagement, they can be difficult to operationalize, particularly within a large-scale research study. The HEALing Communities Study is a large-scale, fast-moving study that offers an opportunity to examine how CBPR principles are demonstrated within community coalition facilitation. This paper utilizes ethnographic data from coalition meetings to examine themes related to barriers and facilitators to operationalizing the CBPR principles during the initial year of coalition formation and EBP selection in Massachusetts.

\section{Methods}

\section{Study Setting and Data Collection Procedures}

Ethnography is a participant observation method that aims to produce highly detailed data describing community experience or respond to a common phenomenon of interest [17]. Ethnographic perspectives are either from an 'emic' insider's view such as that of a participant, or from an 'etic'outsider's view of a researcher who observes but does not participate in the phenomena being studied [18]. In HCS, we used an etic perspective to describe the complex phenomena of coalition building and the use of CBPR principles within study-funded communities to reduce overdose deaths.

For this analysis, ethnographic notes were taken during CTH community coalition meetings across the state between November 2019 (coalition initiation) and December 2020 to cover coalition formation and EBP selection. A total of eight communities were included. These communities represent the Massachusetts HCS communities randomly selected to receive the intervention during the initial time period (Wave 1) and include six urban and two rural communities as defined by the US Census Bureau's American Community Survey (https://data.census.gov/cedsci). Community characteristics of the eight communities by urban-rural status are presented in Table 1 . The eight communities are comprised of just under 350,000 individuals aged 18 or older. In the two rural communities, almost half of the adult 
population was aged 55 or older, while in the six urban communities, the population aged 55 or older represented just over a third of the total adult population. In both rural and urban communities, females comprised approximately half of the adult population. In the urban communities, just over $60 \%$ of the population was white, as compared to over $90 \%$ in the rural communities. Area deprivation indices integrate information on income, education, employment, and housing conditions, with lower scores indicating greater affluence and higher scores indicating greater deprivation, were higher in the urban communities. The large majority (over $85 \%$ in urban communities and over $95 \%$ in rural communities) of adults had received at least a high school diploma. Median household income was almost $\$ 24,000$ lower in urban than in rural communities, and in urban communities, $19 \%$ of households received public assistance, while in the rural communities, households receiving public assistance comprised only $3 \%$ of the population. 


\begin{tabular}{|c|c|c|}
\hline \multirow[t]{3}{*}{ Table 1: Community Characteristics } & \multicolumn{2}{|c|}{ Massachusetts } \\
\hline & Urban & Rural \\
\hline & $\%$ or Est. (SD) & $\%$ or Est. (SD) \\
\hline Number of randomized communities & 6 & 2 \\
\hline Population aged 18 or older, $2019^{1}$ & 300,204 & 49,703 \\
\hline \multicolumn{3}{|l|}{ Distribution of population, aged 18 or older by age ${ }^{1}$} \\
\hline Age 18-34 & 96,604 & 10,966 \\
\hline Age $35-54$ & 95,526 & 14,788 \\
\hline Age $55+$ & 108,074 & 23,949 \\
\hline \multicolumn{3}{|l|}{ Distribution of population, aged 18 or older by sex ${ }^{1}$} \\
\hline Female & 157,433 & 24,427 \\
\hline Male & 142,771 & 25,276 \\
\hline \multicolumn{3}{|l|}{ Distribution of population, aged 18 or older, by race and ethnicity ${ }^{1}$} \\
\hline Black & 38,846 & 806 \\
\hline Hispanic & 43,065 & 1,629 \\
\hline White & 181,730 & 45,791 \\
\hline Other & 36,563 & 1,477 \\
\hline Area Deprivation Index (ADI; scale of $0-1,1$ being highest) ${ }^{2}$ & 37.33 & 20.57 \\
\hline High school education or more, $\%$ of adults aged $25+, 2014-2019^{3}$ & $85.1 \%$ & $95.9 \%$ \\
\hline Median household income, 2014-2019³ & $\$ 57,845$ & $\$ 81,761$ \\
\hline Households receiving public assistance, $\%, 2014-2019^{3}$ & $19.0 \%$ & $3.0 \%$ \\
\hline
\end{tabular}

${ }^{1}$ U.S. Census Bureau. (2021). 2015-2018 American Community Survey 5-year Averages. Retrieved via https://data.census.gov/cedsci on January 27, 2022.

${ }^{2}$ Kind, A. J. H, \& Buckingham W. (2018). Making neighborhood disadvantage metrics accessible: The Neighborhood Atlas. New England Journal of Medicine, 378, 2456-2458. DOI: 10.1056/NEJMp1802313. And: University of Wisconsin School of Medicine Public Health. (2021). 2014-2019 Area Deprivation Index v2.0. Retrieved from https://www.neighborhoodatlas.medicine.wisc.edu/ on January 21, 2021. 
${ }^{3}$ U.S. Census Bureau. (2021). 2014-2019 American Community Survey 5-year Averages. Retrieved via the TidyCensus R package, https://walker-data.com/tidycensus/ on November 1, 2021.

Two members of the Massachusetts HCS Implementation Science research team attended all coalition meetings as participant observers and ethnographers. During coalition meeting introductions, ethnographers introduced themselves as members of the HCS Implementation Science Core who "study the study". The ethnographers attended coalition meetings to observe and take notes on the coalition's process; ethnographers are not a part of community coalitions. Six ethnographers were assigned, in consistent ethnographer dyads, to each coalition for ongoing observation ethnographic data collection. The goal of this approach was to familiarize ethnographers with their assigned coalitions and gain knowledge about the coalition over time.

All six ethnographers were trained in qualitative methods and held degrees in anthropology or sociology, two disciplines that frequently use ethnographic methods. Ethnographers received additional studyspecific training in ethnographic methods from senior researchers with methodological expertise. Training topics included introducing oneself at coalition meetings, engaging in participant observation without 'othering' coalition members, taking field notes, producing a written narrative based on field notes and practicing reflexivity, or acknowledging the experiences and perspectives the researcher is bringing which influence the research environment and work produced $[17,18]$. The ethnographers were also trained by experts in CBPR to identify examples of CBPR principles. The ethnographers used a field note template to promote consistency in data collection across communities and over time. The template included unstructured space for ethnographers to document general meeting observations and a structured table to record examples of, or reflections about, each of the 10 CBPR principles [Appendix 1]. The tables were then used to identify examples that illustrated adherence to or absence of CBPR principles. In addition to full coalition meetings, communities held content-focused subcommittee meetings. Ethnographers were not present during subcommittee meetings and only attended full coalition meetings.

This research was approved by Advarra Inc., the HCS single institutional review board (sIRB: Phoero0038088, Amendment \#03). The IRB approved a waiver of informed consent. The Standards for Reporting Qualitative Research recommendations for reporting on ethnographic approaches in implementation research are used to enhance rigor and transparency in describing the methodological approach and results $[20,21]$.

\section{Data Analysis}

Ethnographic descriptions from the structured CBPR table in the field notes were analyzed using thematic analysis methods to examine presence or absence of CBPR principles during coalition meetings [22]. Two members of the study team independently reviewed the data for each individual principle to identify initial codes. Discussing the set of codes for each CBPR principle was imperative to codebook development and solidifying inclusion and exclusion criteria for each code. Once consensus was reached between the two 
coders and a final codebook was developed, the researchers independently coded the ethnographic tables using the codebook. The coders met regularly to review code application, discuss challenging passages and achieve coding consensus.

Once structured tables containing coalition meeting reflections on CBPR principles were coded, members of the Implementation Science Core discussed preliminary coding to draft themes. The larger research team then met three times to finalize themes related to implementation barriers and facilitators. The research team noted substantial overlapping concepts associated with multiple CBPR principles. While the CBPR principles provided a way to organize data into concepts, the identified themes spanned multiple principles. All coding and qualitative analysis were conducted in NVivo 12.0.

\section{Results}

Over 13 months, there were 101 full coalition meetings across the eight communities, ranging from nine to 16 meetings per community. Most coalitions met once per month for 60 to 120 minutes. During various phases and the emergence of the novel coronavirus 2019 disease (COVID-19), some coalitions opted to meet semi-monthly. All coalitions adopted a virtual meeting format beginning in April 2020 due to the emergence of COVID-19. A total of 429 individuals attended at least one coalition meeting with a range of 23-79 individuals per coalition. The average number of attendees at each coalition meeting per community ranged from 10-29 individuals.

Five central themes emerged from ethnographic analysis that served as facilitators and barriers to realizing specific CBPR principles within the coalition formation and early EBP implementation phases: 1) importance of trust, familiarity and mutual support; 2) magnitude of shared goals; 3 ) power dynamics between research site and community; 4) meeting structure matters; and 5) need to consider the sociopolitical context of the community in planning and implementation. Each theme is described below along with direct statements from the ethnographers and identification of the CBPR principles linked to each theme [Table 2]. Each of these overarching themes sometimes served as a facilitator and other times as a barrier to actualizing the CBPR principles, as described in our characterization of the themes and illustrative statements from the ethnographies. 
Table 2

Themes \& Relevant CBPR Principles

Theme

1. Importance of trust, familiarity and mutual support

\section{Linked Principles}

- Principle 2: Build on strengths and resources within the community

- Principle 3: Facilitate a collaborative, equitable partnership in all phases of the research

- Principle 4: Promote co-learning and capacity-building among all partners

- Principle 5: Integrate and achieve a balance between research and action to mutually benefit all partners

2. Magnitude of shared goals

- Principle 1: Recognize the community as the unit of identity

- Principle 5: Integrate and achieve a balance between research and action to mutually benefit all partners
3. Power dynamics between research site and community

- Principle 3: Facilitate a collaborative, equitable partnership in all phases of the research

- Principle 5: Integrate and achieve a balance between research and action to mutually benefit all partners

- Principle 7: Involves systems development through a cyclical and iterative process

- Principle 8: Disseminate findings to all partners and involve all partners in dissemination
4. Meeting structure matters

- Principle 3: Facilitate a collaborative, equitable partnership in all phases of the research

- Principle 4: Promote co-learning and capacity-building among all partners

- Principle 7: Use of a cyclical and iterative process

- Principle 8: Disseminate findings to all partners and involve all partners in dissemination

5. Need to consider community sociopolitical context
- Principle 6: Emphasize public health problems of local relevance and ecological perspectives that recognized the multiple determinants of health and disease

- Principle 10: Addresses issues of race, ethnicity, racism, and social class and embraces "cultural humility"

\section{Theme 1: Importance of trust, familiarity, and mutual support}

Trust, familiarity, and mutual support among coalition members serves as a facilitator supporting the demonstration of Principles 2, 3, 4 \& 5. 
The coalition is incredibly collaborative and are constantly offering ways to support each other. There is no feeling of competition for funding or resources between the organizations [represented by coalition members]. They appear unified in their goal of supporting their shared community [Community \#4, November 2020].

Working within a familiar and supportive environment fosters collaboration and capacity building among coalition members and enables them to build on their knowledge and strengths, both individually and within their organizations.

This meeting demonstrated how the coalition may be moving from a combative "storming" phase to a more peaceful "norming" phase. As coalition members presented their intervention updates they highlighted how other coalition members had collaborated or contributed to progress. Coalition members demonstrated appreciation for each other's efforts [Community \#3, November 2020].

Similarly, a trusting relationship between the HCS Community Engagement Facilitator and coalition members created space for shared ownership of the work and balanced research and community priorities necessary to implement interventions. Facilitation skills supported the presence of Principle 5 and trusting relationships opened space for feedback from coalition members.

They (CEFs) are truly facilitators... allowing the coalition to take ownership and share updates, while sharing research site updates in clear, accessible language. The participation from various coalition members and the constructive feedback provided to the research site staff shows a clear investment from coalition members in the materials and findings of the study [Community \#6, August 2020].

Another ethnographer noted the facilitators focused on building strong community relationships with coalition members and community organizations, which require expanding coalition membership to bring in new voices and share additional resources:

The coalition members speaking up now were not the ones speaking up in the beginning and were not even present at the table. It is exciting to be hearing from peer recovery coaches, and volunteers not just town officials. The CEF's facilitation skills and local relationships/knowledge are key in this change I believe [Community \#2, May 2020].

Conversely, a lack of trust, familiarity and mutual support served as a barrier to the demonstration of these principles. In the absence of familiarity and trust, coalition members tended to prioritize their own organizations, and they were less engaged in collaboration and/or learning about others and their strengths or focusing on the good of the larger community:

Coalition members listened to each other's ideas and were respectful. However, the very vocal organizations seem to want to prioritize their suggested interventions. Additionally, coalition members still seem to be unfamiliar with one another as observed when [coalition member] referenced [other coalition member] as "the gentleman from [local organization]"; there do not seem to be set standards for referencing other coalition members [Community \#1, July 2020]. 
While some interventions may address and support co-learning, there were instances where organizations were more interested in interventions strictly promoting their own organization:

"The idea of a navigator at each organization who would communicate with other organizations would be a space that facilitated co-learning. However, [local organization] and [local organization] are struggling with how to promote capacity building at both partners instead of just advocating for their own organization." [Community \#3, August 2020].

\section{Theme 2: Magnitude of shared goals}

Shared goals among coalition members appeared to be a critical facilitator for the presence of Principles 1 and 5 at coalition meetings. Coalitions with shared goals seemed to instinctively promote the idea of the community as their collective unit of identity and demonstrated openness to changes and true partnership based on coalition and community needs:

"A coalition member shared that sentiment that they want and need feedback from the community and other organizations in order to be as effective as possible in their outreach. They shared that while their agency is housing the position, they are serving the community which was powerful." [Community \#5, December 2020].

A mutual understanding and set of shared goals were also very important between the research site and the coalition in terms of promoting trust. Ethnographers identified this as critical to moving forward:

The research site...explains the expectations of the study from the start of the coalition, the charter outlined the expectations of the coalition membership and provided the members an opportunity to vote on their commitment to the coalition by agreeing to the charter [Community \#2, February 2020].

Conversely, in cases where the coalition did not share goals with the research site, coalition engagement and movement toward the study goals waned:

The coalition currently does not believe that this project is beneficial to them. There is a clear feeling that the work they will be doing is replicating work that is already occurring in the community. The research site is not doing a good job of explaining how the community can use the money from the grant to fill in gaps [Community \#2, February 2020].

\section{Theme 3: Power dynamics between research site and community}

Power dynamics between the research site and coalition members emerged as both a barrier and facilitator to operationalizing Principles 3, 5, 7 and 8. Coalition members displayed stronger engagement when the research site and coalition shared power and space in meetings:

The facilitator does a good job of allowing the coalition to take ownership of several action items and present their own opinions on various barriers and services in the community. The discussions are not 
formally structured but seem to work well for this group; people appear comfortable providing feedback regardless of their organization or expertise. The meeting agenda guides the coalition in terms of expectation and meeting topics but otherwise the conversation flows naturally with some facilitation from [CEF]. [Community \#1, May 2020].

Strategies such as facilitated discussions to uplift coalition members' voices addressed the inherent power imbalance between the coalition and the research site, and encouraged an equitable relationship between the parties. One ethnographer noted,

All participants were asked to introduce themselves and identify their hopes and concerns about the study. This led to an open discussion of shared goals and potential issues from the community perspective. This activity created an environment where community voices were elevated, so that the process was not driven entirely by the research team. All of the hopes/issues were written on a board at the front of the room for everyone to see and reflect on [Community \#4, February 2020].

Power sharing was evident when coalition members serving in champion roles began leading meetings, presenting information to and engaging with their peer coalition members rather than relying on the Community Engagement Facilitator or other study staff to lead this process. In one coalition,

The data and communications champions are taking on a bigger role in presenting updates to the coalition. In these two instances, the community-facing team takes more of a backseat and coalition members are beginning to take on more ownership of these activities. [Community \#6, April 2020].

Power dynamics were also a barrier to Principles $3,5,7$ and 8 . When the research site did not actively include coalition members' feedback and voices, coalition members were less invested in the work. An ethnography notes, "The iterative process was lacking. After learning that several of their selected intervention strategies were not approved by the HCS research team, the coalition did not engage in problem-solving activities. Instead, the meeting had a very negative, demobilizing energy' [Community \#4, July 2020]. Since the research site inherently holds power as the purveyor of study funding, it was important for community-facing teams to address this imbalance and create space for coalition member voices as part of incorporating the CBPR principles, while being transparent about which decisions were in the purview of the coalition, and which were ultimately up to the research site. Coalition members occasionally called attention to the impact of power dynamics between the research site and coalition that highlighted the relationship as a barrier to Principle 5:

There seems to be tension between the research site and coalition members. The breakout room I was in discussed that the coalition feels dictated by [research site] instead of led by the coalition. They explained there is not transparency and clarity between the two parties, and if the community had real ownership of the coalition, they could get this work done more efficiently. [Community \#7, December 2020].

\section{Theme 4: Meeting structure matters}


As a community-engaged study, the coalition meeting structure served to facilitate engagement, collaborative partnerships, and a sense of ownership among coalition members. When meeting time was structured, coalition meetings demonstrated principles 3, 4, 7 and 8 . As described in theme 3 related to power dynamics between research site and community above, having a structure that included content experts on the coalition as champions also helped to navigate the power dynamic between the research site and the coalition. One ethnographer noted how using content champions and creating space for coalition member updates increased engagement and ownership by members:

Coalition members shared updates from the subgroups. Each update was clear and comprehensive. Due to the large amount of discussion that occurred after each subgroup updates, it appears the coalition members are beginning to feel invested and responsible for the progress being made. There is some sense of "ownership" over each of the subgroups, which is shared by several coalition members, but all appear comfortable commenting on the various subgroups' work." [Community \#1, May 2020].

Other examples of intentional meeting structure, such as breakout rooms, also facilitated a sense of ownership among the coalition:

The break-out groups were a great way to start allowing community members to work and learn from each other. The different facilitators in each room provide a different capacity for co-learning. The community faculty member is a skilled facilitator who frames [their] breakout session in a series of questions to receive the most information around the data presented as possible. This promotes colearning as the research site provides the data and the coalition discusses the reality of what the data shows and how useful the data are to the coalition and the work they are trying to accomplish [Community \#8, April 2020].

The structure and use of meeting time were important factors for whether Principles 3, 4, 7 and 8 were demonstrated. In contrast, limitations in meeting structure due to study timeline restrictions posed challenges to engagement. "All updates are followed by discussion to provide feedback and suggestions although this space is not often taken advantage of. Due to the timing challenges experienced in today's coalition meeting, the spaces for discussion did not feel like enough time." [Community \# 1, November 2020]. The COVID-19 pandemic created new structure challenges due to remote meetings. Several ethnographies noted the barriers related to remote meeting structures and how they posed challenges for engagement and decision making: "Had we not been on Zoom, it would have been easier to gage the coalition members (who were off camera) responses" [Community \#5, September 2020]; "The meeting was planned to be shorter in length than normal due to Zoom fatigue" [Community \#6, May 2020]; "It is a bit hard to do consensus on Zoom" [Community \#2, June 2020].

\section{Theme 5: Need to consider community socio-political context}

Coalition meetings often included discussions about ongoing social and political issues such as racial equity, economic stability, stigma, insufficient affordable housing and other factors that impact

Page $14 / 25$ 
community members' access to prevention, harm reduction and treatment for opioid use disorder. Framing opioid use disorder within the existing local community, state and national sociopolitical contexts was highly important to stakeholders. At times, coalition members facilitated conversations around principles 6 and 10, and emphasized the importance of incorporating an equity lens into their work. One ethnographer notes,

In this coalition meeting the CDM shares a PowerPoint with local overdose data. A coalition member is concerned that there is no data for Black Americans being shown in demographics graph. The coalition member shared their concern because they know Hispanic and African American populations are being hurt. She is astonished that there have been zero deaths in two years among African Americans. [Community \#5, June 2020].

Coalition members engaged in conversations that focused on stigma, a lack of affordable housing and the COVID-19 pandemic. These discussions prompted meeting leaders to focus on relevant local public health issues in coalition meetings:

The coalition discussed how stigma prevents many in the community from accessing Naloxone. They also mentioned barriers and regulations that dictate which organizations can distribute Naloxone and how that needs to be tracked. The coalition considered these factors when brainstorming interventions, and they were leaning towards expanding Naloxone. A coalition member shared that their agency has been trying to get NaloxBoxes for a while and COVID-19 put a halt on that effort. They added that it is difficult to get places to put out the boxes in public because of stigma, people say that the boxes 'invite those people here'" [Community \#2, June 2020].

Discussions of stigma and community social context could also facilitate actualization of other CBPR principles such as co-learning and capacity building across stakeholders. For example, one ethnography noted, "There was a lovely example of co-learning in today's meeting when one coalition member shared appropriate language with the coalition encouraging everyone to read through it and think about the language used to discuss Substance Use Disorder" [Community \#2, October 2020].

When discussions about sociopolitical context arose in coalition meetings, ethnographers noted their importance and relation to coalition efforts or community engagement. However, many ethnography tables often stated, "this principle was not discussed during this meeting." Principle 10 related to race, ethnicity and class was discussed less frequently than related to principle 6 , addressing public health issues of relevance, which were naturally addressed due to the study focus. A frequent note in the ethnography about Principle 10 was "this principle was not addressed during this meeting."Typically, ethnographies identified a lack of focus on these principles as appearing due to time constraints, which presented a significant barrier to consistently addressing these issues in coalition meetings. One ethnographer noted the limited time given to these topics in a coalition meeting,

A passionate conversation occurred around the need to address housing stability for individuals with opioid use disorder, particularly in light of COVID-19 and the approaching winter months. While the 
discussion was well-intended and expressed appreciation of interconnections in individual's lives, little progress was made due to time constraints. This issue will continue to be discussed in a smaller group moving forward." [Community \#7, November 2020].

Some coalitions determined that the better way to address these issues were via subcommittees or other small groups focused on race, equity and social justice, and then brought discussions back to the larger coalition once a potential strategy had been identified in the smaller group.

\section{Discussion}

We examined how the CBPR principles were operationalized by community research teams working with eight coalitions established as part of CTH intervention in a study that used facilitation as a primary implementation strategy. Five core themes were observed related to facilitators and barriers to the adoption of CBPR principles. Across the eight communities studied, we found that when trusting relationships existed both among coalition members themselves, as well as between the research site's community team and the coalition members, the principles were likely to be exhibited. Shared goals related to research activities enhanced the use of CBPR principles. Power and power sharing emerged as a key theme, and the extent to which power dynamics were attended to impacted coalition functioning. Meeting structure also impacted the actualization of the CBPR principles. Lastly, it was necessary within the coalition structure to attend to the social context within communities, specifically the intersection of social determinants of health, stigma, and marginalization of people who use drugs. Despite the importance of social context, issues related to race and cultural humility often were not discussed during coalition meetings for a multitude of reasons including time constraints and study deliverables.

Trust served as a facilitator to embodying the CBPR principles. Coalition engagement that involved shared understanding of decision-making power fostered mutual respect and joint problem solving between the research team and coalition members. Our study also found that creating opportunities for coalition member engagement and elevating community expertise was critical for demonstrating the CBPR principles. Trusting relationships between the coalition facilitators and coalition members allowed for candid feedback around the study process. These results are consistent with findings from other studies, such as a study that examined important elements of building early trust between researchers and American Indian communities, which identified the importance of understanding the research, listening to community members, acknowledging the expertise of all partners, and being clear upfront about expectations and intentions [23].

Shared goals encouraged collaboration and ownership among the coalition. This aligns with the literature that highlights the influence of shared goals on a group's decision-making and process [24]. These coalitions were comprised of a broad range of stakeholders, some of whom were new to one another, as well as research staff, uniting over a shared vision facilitated the CBPR principles. The value of shared goals speaks to the importance of the facilitator's role in uniting the group over a shared vision. While 
goal-oriented work engaged partners in this study, previous research has also highlighted that focusing on narrow goals rather than addressing systemic forces can limit ability for change-making $[25,26]$.

Power dynamics between the research site and coalition served as both a facilitator and barrier to the CBPR principles. Community teams who created space for coalition ownership were able to share power within the inherently hierarchical research structure. These facilitation skills allowed those communities to embody the CBPR principles. On the other hand, power dynamics associated with the hierarchical structure led coalition members to be less engaged. Addressing power dynamics is critical for successful CBPR, with some suggesting that the CBPR principles cannot be operationalized unless these power dynamics are identified and addressed during the research process [27]. The power dynamics we found between the research site and the coalition are not surprising for several reasons. These were often new coalitions that needed to boundary span or boundary separate at times, needing to integrate with unfamiliar communities or separate one community from existing cross-community work. The power dynamics within coalitions were also not surprising, given the stages of forming, storming and norming that needed to happen before a coalition could get to the performing stage or the time for identifying and setting up interventions $[28,29]$.

Coalition meetings served as spaces in which community teams were able to operationalize the CBPR principles. Specific aspects of meeting structure, such as smaller groups and content champions, were foundational for actualizing the CBPR principles of recognizing the community as a unit of identity and engaging in co-learning and shared decision-making. However, when meeting structure was impeded by time restrictions, actualizing the principles was more challenging. When coalition meetings were required to move from in-person to remote during the COVID-19 pandemic, there were multiple times where ethnographers noted the challenge of perceiving engagement when participants did not have video cameras on. Community-based organizations, particularly those serving marginalized populations, were extremely impacted by the COVID-19 pandemic [30] and substance use treatment providers and their patients may have been especially affected [31, 32]. Coalition meetings held during the workday likely served as a barrier for engagement as coalition members were also attending to other responsibilities.

Addressing the socio-political community context served as a facilitator for the CBPR principles. Stigma related to substance use emerged as an important community contextual factor. In early coalition formation, stigma toward persons with opioid use disorder and persons using opioids was clearly acknowledged by coalition members as a critical issue. The issue of stigma in sometimes complicated relationship development, particularly related to both the history and structure of research organizations. Stigma serves to marginalize some members of the community whose voices are most needed to inform this work.

Issues of race, ethnicity and cultural humility were less frequently addressed during these early coalition meetings, likely often due to time restraints and competing study priorities, despite the fact that the opioid overdose epidemic disproportionately impacts persons of color and marginalized populations and that these inequities only amplified during the COVID-19 pandemic $[33,34,35,36]$. It was also notable that, 
although several of the communities were comprised of racially and culturally diverse populations, the coalitions were composed predominately of white individuals who often worked for well-established healthcare and treatment providers. Yet coalition members stated their desire to expand the diversity of coalitions, and several coalitions created subcommittees to create space for equity-related work. The need for research to quickly move forward often leads to partnerships being predominantly with larger organizations or institutions that hold power, or allowing these organizations to hold the majority of the power within a coalition. In doing so, there is the risk of further contributing to inequitable dynamics on the coalition, pointing to the critical need for the balance of power and role of the facilitator in both engaging and elevating the voices of those most marginalized as they work to build consensus and engagement within the coalition. However, not addressing these issues serves to perpetuate existing inequities and replicate existing structures that produce inequities. This will be an important challenge and opportunity for the coalitions moving forward.

This paper is not without limitations. First, our work was conducted in one relatively small state and therefore may not be reflective of how coalitions will operate in other states that participate in this study. Second, as with all qualitative data, our findings may not be generalizable to other settings. However, our findings may be transferable to other implementation studies that use a community-engaged facilitation approach and coalition-building as a primary implementation strategy. Moreover, our findings regarding how CBPR principles can be used to address a highly stigmatized issue and conform to a rapid study timeline may be transferrable to other CBPR studies. Third, our study included data only from full coalition meetings, and we were unable to capture smaller subcommittee meetings which may have been facilitated differently by community-facing teams or have different dynamics due to smaller numbers of individuals participating. Since the full coalition meeting includes both coalition members and study staff, the dynamic and discussion is inherently different from smaller, more focused meetings. In practice, there were also numerous planning and coaching meetings with individual community members to support them to prepare for larger coalition meetings. Some communities also initiated subcommittees related to equity and racial/social justice that may also have addressed issues of race, ethnicity and cultural humility more frequently. However, while we were unable to obtain data from smaller meetings, we were able to obtain data from a large number of meetings that were therefore likely reflective of the overall tenor of the coalition process. Fourth, like all ethnographic data, the findings are from the perspective of ethnographers. We took an etic perspective; therefore, our findings represent the outsider/observer perspective rather than the insider/participant perspective. While we do not have the insider perspective, an important strength is that our data collection was direct rather than reported at a later time point via interviews. This enables us to partially overcome concerns related to misremembering or biased self-reporting. This perspective is also useful for understanding how people delivering or receiving an intervention behave in real life. Fifth, for this analysis we solely coded the structured tables of CBPR principles rather than the entire set of ethnographic field notes taken at each meeting. Given the focused nature of this analysis on themes related to the presence and absence of these principles, coding the entire ethnography may yield additional insights. 
It is important to note that a few months into coalition formation and facilitated coalition meetings, the COVID-19 pandemic occurred. At this time, meetings that had begun in person transitioned to video and telephonic meetings. Early on in this process, many participants experienced technological challenges that may have impacted facilitation and interactions. For example, sometimes facilitators and/or community members were present on audio but not video formats. Also during this time, some coalition members lost their jobs while others necessarily prioritized responsibilities to their organizations, which may have led to less engagement during this extended pandemic. However, the coalitions and the work of standing up interventions continued despite the many challenges, and the focus on the socio-political context became potentially even more important in light of the challenges of COVID-19 and its impact on highly stigmatized populations. While COVID-19 likely challenged the use of the CBPR principles, this natural experiment provides an important lesson in coalition formation and ability to perform for community good even in the most difficult times. It is very likely that operationalizing the CBPR principles played an important role in coalitions being able to continue strong functioning during this time.

\section{Conclusions}

By using ethnography as a new form of analysis for CBPR principles, our results provide important insights about a community-engaged approach along with facilitators and barriers to utilizing the CBPR principles. Our findings also highlight the importance of facilitating a partnership that fosters co-learning and integrating community needs as well as the imperatives of the research and the need to acknowledge issues of stigma, socio-political context and social determinants of health as important to representation. Prior studies underscore the importance of all stages of the community-academic partnership [37]. While understanding partnership development and early formation to implement the EBPs is vital, future research on the HCS coalitions should also consider how partnerships morph over time, including examining the active EBP implementation phase and the concluding phase of the active community engagement activities. It is notable, although perhaps not surprising, that CBPR Principle 9, establishing a long-term commitment to the process, was not noted by the ethnographers and not addressed by the community-facing teams during early coalition formation. However, this longer term commitment to the process will be important to assess over time once the study period ends to understand how coalitions both sustain the intervention and maintain their new partnerships separate from the academic partner in order to continue to address the multifaceted and complicated opioid overdose crisis.

\section{Abbreviations}

CBPR

Community Based Participatory Research

CDM

Community Data Manager

CE

Community Engagement 
CEF

Community Engagement Facilitator

CEnR

Community Engaged Research

EBP

Evidence-based Practice

HCS

HEALing Communities Study

HEALing Communities Study

Helping to End Addiction Long-term Communities Study

MOUD

Medication for Opioid Use Disorder

OEND

Overdose Education and Naloxone Distribution

ORCCA

Opioid Reduction Continuum of Care Approach

\section{Declarations}

Ethics approval and consent to participate: This research is approved by Advarra Inc., the HCS single institutional review board (sIRB: Phoero0038088, Amendment \#03).

Consent for publication: Not applicable.

Competing interests: The authors declare that they had no competing interests.

Funding: This research was supported by the National Institutes of Health and the Substance Abuse and Mental Health Services Administration through the NIH HEAL (Helping to End Addiction Long-term ${ }^{\text {SM}}$ ) Initiative under award numbers UM1DA049394, UM1DA049406, UM1DA049412, UM1DA049415, UM1DA049417 (ClinicalTrials.gov Identifier: NCT04111939). This study protocol (Pro00038088) was approved by Advarra Inc., the HEALing Communities Study single Institutional Review Board. We wish to acknowledge the participation of the HEALing Communities Study communities, community coalitions, and Community Advisory Boards and state government officials who partnered with us on this study. The content is solely the responsibility of the authors and does not necessarily represent the official views of the National Institutes of Health, the Substance Abuse and Mental Health Services Administration or the NIH HEAL Initiative $\mathrm{SM}$.

\section{Author Statement:}

Mari-Lynn Drainoni: Conceptualization, Methodology, Formal Analysis, Writing- Original Draft, Supervision 
Galya Walt: Conceptualization, Methodology, Formal Analysis, Investigation, Writing- Original Draft, Review \& Editing

Linda Sprague Martinez: Conceptualization, Writing- Review \& Editing, Supervision

Rebecca Smeltzer: Writing- Review \& Editing

Savanna Santarpio: Writing- Review \& Editing

Rosie Munoz-Lopez: Writing- Review \& Editing

Craig McClay: Writing- Review \& Editing

Lauren Keisling: Writing- Review \& Editing

Aumani Harris: Writing- Review \& Editing

Faizah Gillen: Writing- Review \& Editing

Valerie El-Alfi: Writing- Review \& Editing

Erika L. Crable: Investigation, Writing- Review \& Editing

Jane F. Carpenter: Writing- Review \& Editing

Laura K. Barkowski: Formal Analysis, Investigation, Writing- Review \& Editing

Tracy A. Battaglia: Conceptualization, Writing- Review \& Editing, Supervision

Acknowledgements: The authors would like to thank Carol Bigsby for her assistance in preparing the manuscript for publication.

\section{Availability of Data and Materials:}

The datasets generated and/or analysed during the current study are not publicly available due to the nature of ethnographic data and concerns about identifiability.

Authors' information:

Affiliations:

Boston University, Boston, MA

Mari-Lynn Drainoni

Linda Sprague Martinez 
Tracy A. Battaglia

\section{Boston Medical Center, Boston, MA}

Galya Walt

Rebecca Smeltzer

Savanna Santarpio

Rosie Munoz-Lopez

Craig McClay

Lauren Keisling

Aumani Harris

Faizah Gillen

Valerie El-Alfi

Jane F. Carpenter

Laura K. Barkoswki

Tracy A. Battaglia

University of California San Diego, San Diego, CA

Erika L. Crable

Corresponding author:

Mari-Lynn Drainoni, PhD, MEd

Section of Infectious Diseases, Boston University School of Medicine

801 Massachusetts Avenue, Room 2014

Boston, MA 02118 USA

Telephone: 617-414-5287

Email: drainoni@bu.edu

\section{References}


1. Centers for Disease Control and Prevention [Internet]. Drug Overdose Deaths in the U.S. Up 30\% in 2020. Atlanta (GA): Centers for Disease Control and Prevention (US); 2021 July [cited 2021 Dec 28]. Available from: https://www.cdc.gov/nchs/pressroom/nchs_press_releases/2021/20210714.htm

2. Macmadu A, Batthala S, Correia Gabel AM, Rosenberg M, Ganguly R, Yedinak JL, et al. Comparison of characteristics of deaths from drug overdose before vs during the COVID-19 pandemic in Rhode Island. JAMA Netw Open. 2021;4(9):e2125538. https://doi.org/10.1001/jamanetworkopen.2021.25538

3. Patel I, Walter LA, Li L. Opioid overdose crises during the COVID-19 pandemic: implication of health disparities. Harm Reduct J. 2021;18(1):89. https://doi.org/10.1186/s12954-021-00534-z

4. Minkler M., Wallerstein N. Community based participatory research for health: process to outcomes. 2nd ed. San Francisco, CA: Jossey Bass; c2008.

5. Key KD, Furr-Holden D, Lewis EY, Cunningham R, Zimmerman MA, Johnson-Lawrence $V$, et al. The continuum of community engagement in research: a roadmap for understanding and assessing progress. Prog Community Health Partnersh. 2019;13(4):427-434.

https://doi.org/10.1353/cpr.2019.0064

6. Wallerstein N, Duran B. The theoretical, historical, and practice roots of CBPR. In: Minkler N, Wallerstein $\mathrm{N}$, editors. Community-based participatory research for health: from process to outcomes. (pp. 25-46). San Francisco, CA: John Wiley \& Sons; c2008. P. 25-46.

7. Israel B, Schulz A, Parker E, Becker A., Allen A, Guzman R III, et al. Critical Issues in Developing and Following CBPR Principles. In: Wallerstein N, Duran B, Oetzel J, Minkler N, editors. Community-based participatory research for health: Advancing social health equity. San Francisco, CA: John Wiley \& Sons; c2018. p. 31-43.

8. Collins SE, Clifasefi SL, Stanton J, The LEAP AB, Straits K, Gil-Kashiwabara E, et al. Communitybased participatory research (CBPR): towards equitable involvement of community in psychology research. Am Psychol. 2018;73(7):884-98. https://doi.org/10.1037/amp0000167

9. Burke JG, Hess S, Hoffmann K, Guizzetti L, Loy E, Gielen A, et al. Translating community-based participatory research principles into practice. Prog Community Health Partnersh. 2013;7(2):115122. https://doi.org/10.1353/cpr.2013.0025

10. Oetzel JG, Wallerstein N, Duran B, Sanchez-Youngman S, Nguyen T, Woo K, et al. Impact of participatory health research: a test of the community-based participatory research conceptual model. Biomed Res Int. 2018;2018:7281405. https://doi.org/10.1155/2018/7281405

11. Chandler RK, Villani J, Clarke T, McCance-Katz EF, Volkow ND. Addressing opioid overdose deaths: the vision for the HEALing communities study. Drug Alcohol Depend. 2020; 217:108329. https://doi.org/10.1016/j.drugalcdep.2020.108329

12. HEALing Communities Study Consortium. The HEALing (helping to end addiction long-term ${ }^{S M}$ ) communities study: protocol for a cluster randomized trial at the community level to reduce opioid overdose deaths through implementation of an integrated set of evidence-based practices. Drug Alcohol Depend. 2020;217:108335. https://doi.org/10.1016/j.drugalcdep.2020.108335 
13. Sprague Martinez L, Rapkin BD, Young A, Freisthler B, Glasgow L, Hunt T, et al. Community engagement to implement evidence-based practices in the HEALing communities study. Drug Alcohol Depend. 2020;217:108326. https://doi.org/10.1016/j.drugalcdep.2020.108326

14. Winhusen T, Walley A, Fanucchi LC, Hunt T, Lyons M, Lofwall M, et al. The opioid-overdose reduction continuum of care approach (ORCCA): evidence-based practices in the HEALing communities study. Drug Alcohol Depend. 2020;217:108325. https://doi.org/10.1016/j.drugalcdep.2020.108325

15. Lefebvre RC, Chandler RK, Helme DW, Kerner R, Mann S, Stein MD, et al. Health communication campaigns to drive demand for evidence-based practices and reduce stigma in the HEALing communities study. Drug Alcohol Depend. 2020;217:108338.

https://doi.org/10.1016/j.drugalcdep.2020.108338

16. Wallerstein N, Minkler M, Carter-Edwards L, Avila M, Sánchez V. Improving health through community engagement, community organization, and community building. In: Glanz K, Rimer BK, Viswanath VK, editors. Health behavior: theory, research, and practice. Hoboken, NJ, US: Jossey-Bass/Wiley; c2015. p. 277-300.

17. Green J, Thorogood N. Qualitative methods for health research. 3rd ed. Thousand Oaks, CA: SAGE Publications Inc.; c2014.

18. Hall WA, Callery P. Enhancing the rigor of grounded theory: incorporating reflexivity and relationality. Qual Health Res [Internet]. 2001;11(2):257-72. Available from: http://dx.doi.org/10.1177/104973201129119082

19. Reeves S, Kuper A, Hodges BD. Qualitative research methodologies: ethnography. BMJ. 2008;337. https://doi.org/10.1136/bmj.a1020

20. Gertner AK, Franklin J, Roth I, Cruden GH, Haley AD, Finley EP, et al. A scoping review of the use of ethnographic approaches in implementation research and recommendations for reporting. Implement Res Pract. 2021;2. https://doi.org/10.1177/2633489521992743

21. O'Brien BC, Harris IB, Beckman TJ, Reed DA, Cook DA. Standards for reporting qualitative research: a synthesis of recommendations. Acad Med. 2014;89(9):1245-1251. https://doi.org/10.1097/ACM.0000000000000388

22. Ryan GW, Bernard HR. Techniques to identify themes. Field methods. 2003;15(1):85-109. https://doi.org/10.1177/1525822X02239569

23. Christopher S, Watts V, McCormick AKHG, Young S. Building and maintaining trust in a communitybased participatory research partnership. Am J Public Health [Internet]. 2008 Aug 1 [cited 2021 Nov 30];98(8):1398-406. https://ajph.aphapublications.org/doi/10.2105/AJPH.2007.125757

24. Gilson LL, Shalley CE. A little creativity goes a long way: An examination of teams' engagement in creative processes. J Manage. 2004;30(4):453-70. https://doi.org/10.1016/j.jm.2003.07.001

25. Ishimaru AM. Rewriting the rules of engagement: Elaborating a model of district-community collaboration. Harv Educ Rev. 2014;84(2):188-216.

26. McLaughlin MW. The Rand Change Agent Study: Ten Years Later. In: Ogden AR, editor. Education Policy Implementation. Albany, NY: State University of New York Press; 1991. p. 143-155. 
27. Muhammad M, Wallerstein N, Sussman AL, Avila M, Belone L, Duran B. Reflections on researcher identity and power: the impact of positionality on community based participatory research (CBPR) processes and outcomes. Crit Sociol. 2015;41(7-8):1045-1063.

https://doi.org/10.1177/0896920513516025

28. McGinnis PB, Hunsberger M, Davis M, Smith J, Beamer BA, Hastings DD. Transitioning from CHIP to CHIRP: blending community health development with community-based participatory research. Fam Community Health. 2010;33(3):228-237. https://doi.org/10.1097/FCH.0b013e3181e4bc8e

29. Tuckman BW. Developmental sequence in small groups. Psychol Bull. 1965;63:384-399. https://doi.org/10.1037/h0022100

30. Pinto RM, Park S. COVID-19 pandemic disrupts HIV continuum of care and prevention: Implications for research and practice concerning community-based organizations and frontline providers. AIDS Behav. 2020;24(9):2486-2489. https://doi.org/10.1007/s10461-020-02893-3

31. Pagano A, Hosakote S, Kapiteni K, Straus ER, Wong J, Guydish JR. Impacts of COVID-19 on residential treatment programs for substance use disorder. J Subst Abuse Treat. 2021;123:108255. https://doi.org/10.1016/j.jsat.2020.108255

32. Rosca P, Shapira B, Neumark Y. Isolating the isolated: Implications of COVID-19 quarantine measures on in-patient detoxification treatment for substance use disorders. Int J Drug Policy. 2020;83:102830. https://doi.org/10.1016/j.drugpo.2020.102830

33. Cano M. Racial/ethnic differences in US drug overdose mortality, 2017-2018. Addict Behav 2021;112:106625. https://doi.org/10.1016/j.addbeh.2020.106625

34. James K, Jordan A. The Opioid Crisis in Black Communities. J Law Med Ethics. 2018;46(2):404-21. https://doi.org/10.1177/1073110518782949

35. Lippold, KM, Jones CM, Olsen EO, Giroir BP. Racial/ethnic and age group differences in opioid and synthetic opioid-involved overdose deaths among adults aged $\geq 18$ years in metropolitan areas United States, 2015-2017. MMWR. 2019;68(43):967-973. https://doi.org/10.15585/mmwr.mm6843a3

36. Ochalek TA, Cumpston KL, Wills BK, Gal TS, Moeller FG. Nonfatal opioid overdoses at an urban emergency department during the COVID-19 pandemic. JAMA. 2020;324(16):1673-1674. https://doi.org/10.1001/jama.2020.17477

37. Dill LJ, Gousse Y, Huggins K, Fraser MA, Browne RC, Stewart M, et al. Adjournment in community HIV prevention: Exploring transitions in community-academic partnerships. Health Promot Pract. 2020;21(4):544-51. https://doi.org/10.1177/1524839919839361

\section{Supplementary Files}

This is a list of supplementary files associated with this preprint. Click to download.

- CoalitionBuildingCBPRManuscriptAppendix1.docx 